\title{
The Concept of Vitamin D Sufficiency and Insufficiency in Graves' Disease
}

Latif $Z^{\mathrm{a}}$

\begin{abstract}
Graves' disease (GD) is an autoimmune disorder of the thyroid gland resulting from dysregulation of the immune system against this important endocrine gland. The disease is characterized by hyperthyroidism and presence of circulation auto antibody which stimulates the Thyroid Hormone Receptor(TSHR). The auto antibody is also responsible for goiter and other extra-thyroidal manifestation like ophthalmic, dermatological and others. Vitamin D (Vit.-D) deficiency is reported to link with variety of autoimmune diseases. However the relation between thyroid autoimmunity of Graves'disease and Vitamin D deficiency is still not very clear. But in some studies ${ }^{10-12}$, it was found that there is strong correlation between thyroid autoimmunity and vitamin $D$ $-1,25-(\mathrm{OH})$ deficiency in newly diagnosed GD. It was found $<15 \mathrm{pmol} / \mathrm{L}$. In one study it was found $<12.5 \mathrm{pmol} /$ L. According to definition of insufficiency or deficiency most of the Author agreed to describe insufficiency when Vit D level is less than 30pmol/L and deficient when less than $20 \mathrm{pmol} / \mathrm{L}$. Vitamin D Receptor gene polymorphism is also associated with increased risk of Graves'(4D) disease and higher volume of goiter. In other studies it was found that, the increased thyroid antibody titer was associated with Vitamin D deficiency. Some other studies showed that, treatment with Vitamin D in GD there is significant early improvement of the diseases. Meta-analysis showed low Vitamin D was also associated with higher risk of development of GD. Moreover, hyperthyroidism leading to osteoporosis in GD is also associated with low Vitamin D level and improves by treatment with Vitamin D. However, whether Vitamin D deficiency or insufficiency favor's the onset of GD or supplementation of Vitamin D has any beneficial effect, needs further research.
\end{abstract}

Key word: Autoimmune disorder, Graves' Disease, Thyroid antibody, Thyroid Hormone Receptor, Vitamin D Deficiency.

(BIRDEM Med J 2017; 7(2): 160-163)

\section{Introduction}

Vitamin D deficiency is a common disorder which is known to be associated with increase cardiovascular risk, cancer and even Infection. ${ }^{1}$ Less exposure to sunlight, limited physical activity and even obesity may lead to a low level of Vitamin D in body. ${ }^{2}$ Supplements of Vitamin D reduce these morbidities and also reduce the overall incidence of all cause of mortality. Deficiency of Vitamin D also known to increase the incidence of auto immune disorders like Type 1 diabetes, rheumatoid arthritis and systemic lupus erythematosus (SLE). ${ }^{3}$ Animal studies has shown that there is a

Author Information

a. Professor Zafar Ahmed Latif, Professor of Endocrinology, BIRDEM General Hospial, Dhaka

Address of Correspondence: Professor Zafar Ahmed Latif, Director, BIRDEM Academy, E-mail: zafarlatif2011@yahoo.com

Received: January 05, 2016

Accepted: February 28, 2017 relationship of Vitamin D deficiency with autoimmune thyroid disorders and it is also shown that Vitamin D supplementation with cyclosporine shows reductions of symptoms and effectively prevent the induction of autoimmune thyroiditis. Few studies in human also explored the impact of Vitamin D deficiency on autoimmune thyroiditis where the level of Vitamin D was found to be lower in patients with autoimmune thyroiditis compared with normal healthy volunteers. The same difference of Vitamin D was found in Garves' Disease when compared to (non- AITDs) non-auto immune thyroid disprders eg. toxic nodular goiter. Another study from India showed a weak connection between Vitamin D level and AITDs. Another Indian study also found the correlation between the levels of Vitamin D in AITDs like Hashimoto's thyroiditis and Graves' disease and found significantly low level of Vitamin D in patients with AITDs that were related to the presence of anti-thyroid antibodies and abnormal 
thyroid function tests, suggesting the involvement of vitamin $\mathrm{D}$ in the pathogenesis of AITDs and the advisability of supplementation.

This review will focus on relation of Vitamin D with Graves' Disease immune Thyroid disease) and the role of Vitamin D supplementation as a therapy of Graves' Disease.

\section{Epidemiology}

GD is the most common form of thyrotoxicosis, which may occur at any age but mostly from 20-40 years of age. Prevalence of hyperthyroidism in the general population is $1.2 \%$ among which $0.7 \%$ subclinical hyperthyroidism and $0.4 \%$ Graves' Disease - most common etiology but there is overlap with the subclinical group. Graves' Disease is more common in females than male in 7:1 ratio. ${ }^{4}$

\section{Vitamin D and Thyroid Autoimmune disease}

The molecular mechanism by which vitamin D exerts its action seems to be mediated by its binding tovitamin D-binding proteins receptor (VDR), an intracellular receptor belonging to the steroid/thyroid nuclear receptor family, expressed by human immune cells, such as macrophages, dendritic cells and $\mathrm{T}$ and $\mathrm{B}$ lymphocytes. Vitamin D central target are dendritic cells (DCs). ${ }^{5} 1,25(\mathrm{OH}) 2 \mathrm{D} 3$ inhibitsd DC maturation as well as production of DC-derived cytokines, such as interleukin (IL)-12 and IL-23. In this way, 1,25 $(\mathrm{OH}) 2 \mathrm{D} 3$ alters the Th balance and causes the differentiation of the $\mathrm{T}$ cells in a T-helper 2 phenotype, rather than in T helper 1 (Th1) and Th17 phenotype. DC-derived IL-10 release was enhanced, promoting its tolerogenic properties. ${ }^{6} 1,25(\mathrm{OH}) 2 \mathrm{D} 3$ decreased the production of inflammatory Th1 cytokines such as IL2 and interferon-ã, which promote cell-mediated cytotoxicity leading to thyroid destruction in $\mathrm{HT}^{7}{ }^{7}$ In this regards it can be said that the suppression of Th1 response by Vitamin D may counteract the onset of Graves' Disease, as Th1 dominance seems to be involved in the induction of the disease. ${ }^{8}$ Many studies highlighted the role of vitamin $\mathrm{D}$ in the development of thyroid autoimmune diseases (AITD), which has been also demonstrated in animal models. ${ }^{9}$

In human studies on the association of vitamin D levels with antithyroid antibodies, lower 25(OH)D levels were found in subjects with higher levels of antithyroid antibodies compared with those who were negative for thyroid autoimmunity. ${ }^{10}$ Vitamin D deficiency has been diagnosed more frequently in AITD subjects, especially in those affected by Hashimoto's thyroiditis, than in healthy subjects. ${ }^{11}$ In female patients with newly onset GD, a significant relationship of low levels of $25(\mathrm{OH}) \mathrm{D}$ with increased thyroid volume was observed, suggesting that vitamin $\mathrm{D}$ status may be involved in the pathogenesis of the disease. ${ }^{12}$ At the same time there are some studies which failed to find any relationship between Vitamin D and AITDs. ${ }^{13}$

Most of the study found a primary role of vitamin D deficiency in the pathogenesis of AITD and GD. Vitamin $\mathrm{D}$ found to be able to trigger both innate and adaptive immune responses and to switch the immune system in a tolerogenic sense, avoiding the autoimmune response. However, further studies are needed to determine whether Vitamin D insufficiency is a key factor in the pathogenesis of GD or, rather, a consequence of the disease.

\section{Vitamin D supplementation in thyroid diseases}

Vitamin D is currently being prescribed for the patient with disease condition related to Vitamin D insufficiency. Accordingly to United Stats (US) Endocrine Society, less than $20 \mathrm{ng} / \mathrm{ml}(50 \mathrm{nmol} / \mathrm{l})$ of $25(\mathrm{OH}) \mathrm{D}$ can be consider as vitamin D insufficiency. ${ }^{14}$ $25(\mathrm{OH}) \mathrm{D}$ is considered as the best indicator for monitoring vitamin $\mathrm{D}$ levels because this is the major circulating form of vitamin $\mathrm{D}$, with a half-life of 2-3 weeks. Regarding screening for vitamin D deficiency, the US Endocrine Society guideline recommends it in individuals with specific conditions, who are at risk for this deficiency. None of potential thyroid diseases are included in the list of these conditions. ${ }^{14}$ According to the US Endocrine Society guideline, all adults require at least 600-800 IU daily dietary intake of vitamin D. In the case of deficiency or insufficiency, supplementation is needed and either vitamin D2 or vitamin D3 can be used. Adults who are vitamin Ddeficient should be treated with $50000 \mathrm{IU}$ of vitamin D2 or vitamin D3 once a week for 8 weeks or its equivalent of $6000 \mathrm{IU}$ of vitamin D2 or vitamin D3 daily to achieve a blood level of $25(\mathrm{OH}) \mathrm{D}$ above $30 \mathrm{ng} / \mathrm{ml}$. Supplementation should be continued by a maintenance therapy of 1500-2000 IU daily. ${ }^{14}$ 
Although vitamin $\mathrm{D}$ has been shown to have a potential beneficial role in the treatment of various autoimmune diseases, ${ }^{15}$ the debate surrounding recommendations for vitamin D supplementation in autoimmune disease is still open.

Considering the current available data along with the minimal side effects careful vitamin D supplementation could be considered as a potential tool for the treatment of patients with autoimmune thyroid disease like Graves' Disease.

\section{Discussion}

There are many confounding factors able to influence vitamin $\mathrm{D}$ concentration that have not been properly considered and that can affect the relationship between vitamin D levels and thyroid diseases like GD. Vitamin D metabolism may be influenced by exposure to sunlight, use of sunscreen, naturally dark skin tone, season, geographic latitude, clothing and institutionalization. ${ }^{16}$ Increase air pollution might have an effect on the quality of sunlight and may reduce the production of $25(\mathrm{OH}) \mathrm{D}$ in the body. Other physiological factors like age, body mass index, extracellular volume and vitamin $\mathrm{D}$ binding globulin concentration and affinity can also influence the concentration of Vitamin $\mathrm{D}$ in the body. The racial difference in Vitamin D metabolism is also an important factor and need to be considered as the mean levels of total $25(\mathrm{OH}) \mathrm{D}$ are usually lower in blacks than in whites with a low level of Vitamin D binding globulin, which makes the overall bioavailable $25(\mathrm{OH}) \mathrm{D}$ more or less similar in both races. ${ }^{17}$

In the view of above issues, further large scale clinical studies are needed to confirm the relationship between Vitamin D sufficiency \& insufficiency in Graves' Disease and in other autoimmune thyroid diseases.

\section{Conclusion}

Although several observational studies support the role of vitamin D in the pathogenesis of Graves' Diseases and confirms that Vitamin D seems to inhibit inflammatory responses in human thyroid cells and Tcell production of cytokine leading to the production of TSH receptor autoantibodies, but no guidelines are currently available for or against recommending vitamin D supplementation in the prevention or therapy of
Graves' disease. However, inspite of the different results in different intervention studies, further clinical trials are needed before evidence-based recommendations can be made wheather Vitamin D can play a role in Graves' Disease and other AITDs.

Declaration: This paper was presented in the 33rd World Congress of Internal medicine, Bali, Indonesia, 2016 as invited speech.

\section{References}

1. Mandarino N. Is Vitamin D Deficiency a New Risk Factor for Cardiovascular Disease? Open Cardiovasc Med J 2015; 9: 40-49.

2. Chapuy MC, Schott AM, Garnero P, Hans D, Delmas PD, Meunier PJ. Healthy elderly French women living at home have secondary hyperparathyroidism and high bone turnover in winter: EPIDOS Study Group. J Clin Endocrinol Metab 1996;81: 1129-33

3. Song Y, Wang L, Pittas AG, Del Gobbo LC, Zhang C, Manson JE et al. Blood 25-hydroxy vitamin D levels and incident type 2 diabetes: a meta-analysis of prospective studies. Diabetes Care 2013; 36: 1422-28

4. Cooper GS, Stroehla BC. The epidemiology of autoimmune diseases. Autoimmun Rev 2003; 2: 119-25.

5. Baeke F, Etten EV, Overbergh L, Mathieu C. Vitamin D3 and the immune system: maintaining the balance in health and disease. Nutr Res Rev 2007; 20: 106-18.

6. Mathieu C. Vitamin D and the immune system: getting it right. IBMS BoneKEy 2011; 8: 178-86.

7. Baeke F, Takiishi T, Korf H, Gysemans C, Mathieu C. Vitamin D: modulator of the immune system. Curr Opin Pharmacol 2010; 10: 482-96.

8. Inukai Y, Momobayashi A, Sugawara N, Aso Y. Changes in expression of T-helper (Th) 1 - and Th2-associated chemokine receptors on peripheral blood lymphocytes and plasma concentrations of their ligands, interferon-inducible protein10 and thymus and activation-regulated chemokine, after antithyroid drug administration in hyperthyroid patients with Graves' disease. Eur J Endocrinol 2007; 156:623-30.

9. Misharin A, Hewison M, Chen CR, Lagishetty V, Aliesky HA, Mizutori Y et al. Vitamin D deficiency modulates Graves' hyperthyroidism induced in BALB/c mice by thyrotropin receptor immunization. Endocrinology 2009; 150: 1051-60.

10. Shin DY, Kim KJ, Kim D, Hwang S, Lee EJ. Low serum vitamin $\mathrm{D}$ is associated with anti-thyroid peroxidase antibody in autoimmune thyroiditis. Yonsei Med J 2014; 55: 476-81.

11. Kivity S, Agmon-Levin N, Zisappl M, Shapira Y, Nagy EV, Dankó K et al. Vitamin D and autoimmune thyroid diseases. Cell Mol Immunol 2011; 8: 243-47. 
12. Yasuda T, Okamoto Y, Hamada N, Miyashita K, Takahara M, Sakamoto F et al. Serum vitamin D levels are decreased and associated with thyroid volume in female patients with newly onset Graves' disease. Endocrine 2012; 42: 739-41.

13. Chailurkit LO, Aekplakorn W, Ongphiphadhanakul B. High vitamin D status in younger individuals is associated with low circulating thyrotropin. Thyroid 2013; 23: 25-30.

14. Holick MF, Binkley NC, Bischoff-Ferrari HA, Gordon CM, Hanley DA, Heaney RP et al. Endocrine Society. Evaluation, treatment, and prevention of vitamin D deficiency: an Endocrine Society clinical practice guideline. J Clin Endocrinol Metab 2011; 96: 1911-30.
15. Agmon-Levin N, Theodor E, Segal RM, Shoenfeld Y. Vitamin $\mathrm{D}$ in systemic and organ-specific autoimmune diseases. Clin Rev Allergy Immunol 2013; 45: 256-66.

16. Brannon PM, Yetley EA, Bailey RL, Picciano MF. Summary of roundtable discussion on vitamin D research needs. Am J Clin Nutr 2008; 88: 587-92.

17. Powe CE, Evans MK, Wenger J, Zonderman AB, Berg AH, Nalls $M$ et al. Vitamin D-binding protein and vitamin D status of black Americans and white Americans, N Engl J Med 2013; 369: 1991-2000.

18. Xu M, Cao B, Yin J, Wang D, Chen K, Luet Q al. Vitamin D and Graves' Disease: A Meta-Analysis Update, Nutrients 2015; 7: 3813-27. 